\title{
Minimally invasive facial rejuvenation combining thread lifting with liposuction: A clinical comparison with thread lifting alone
}

\author{
Kwang Il Bae ${ }^{1}$, Dong Gil Han ${ }^{1}$, \\ Sung-Eun Kim ${ }^{1}$, Young Bae Lee ${ }^{2}$ \\ ${ }^{1}$ Department of Plastic and \\ Reconstructive Surgery, School of \\ Medicine, Catholic University of Daegu, \\ Daegu, ${ }^{2}$ Lift Plastic Clinic, Daegu, Korea
}

This article was presented as a free paper at the PRS Korea 2017 on November 12, 2017, in Seoul, Korea

\begin{abstract}
Background Barbed thread lifting is known to be a minimally invasive procedure compared with standard incisional surgery for facial rejuvenation. However, some studies have pointed out that the effects of thread lifting do not last long and disappear after several months. Therefore, the aim of this study was to evaluate the efficiency and duration of anchoring-type absorbable thread lifting combined with liposuction for facial rejuvenation.

Methods A retrospective review of 27 cosmetic patients who underwent absorbable barbed thread lifting with liposuction between July 2013 and December 2015 was undertaken. The efficiency of facial rejuvenation was evaluated through a patient satisfaction score and the global aesthetic improvement scale (GAIS).

Results Subjects were divided into two groups: thread lifting only and thread lifting combined with liposuction. The mean follow-up period of each group was 8.5 and 9.3 months, respectively. In the thread lifting-only group, the average patient satisfaction score was 2.36 (1=unsatisfied to 4=very satisfied), and the average GAIS score was 2.54 (1=very much improved to $5=$ worsen). In the thread lifting with liposuction group, the average patient satisfaction score was 3.06, and the average GAIS score was 1.85 . Conclusions Thread lifting combined with liposuction showed better patient satisfaction and GAIS results than thread lifting alone, even over a long follow-up period.
\end{abstract}

Keywords Suture anchors / Lipectomy / Rejuvenation

\section{INTRODUCTION}

Facial aging is characterized by a progressive increase in the laxity of skin and soft tissue, superficial skin changes, and volume loss. Due to advances in the understanding and comprehension of these processes, many operations and procedures for facial rejuvenation

Received: Dec 6, 2018 Revised: Feb 18, 2019 Accepted: Mar 22, 2019 Correspondence: Dong Gil Han Department of Plastic and Reconstructive Surgery, School of Medicine, Catholic University of Daegu, 33 Duryugongwon-ro 17-gil, Nam-gu, Daegu 42472, Korea Tel: +82-53-650-4474, Fax: +82-53-650-4584, E-mail: dghan1001@cu.ac.kr

Copyright @ 2019 The Korean Society for Aesthetic Plastic Surgery.

This is an Open Access article distributed under the terms of the Creative Commons Attribution Non-Commercial License (http://creativecommons.org/licenses/by-nc/4.0/) which permits unrestricted non-commercial use, distribution, and reproduction in any medium, provided the original work is properly cited. www.e-aaps.org have been devised $[1,2]$.

However, patients now prefer minimally invasive procedures, which have few complications, allow a rapid recovery, and enable patients to quickly return to their daily activities [3]. Thread lifting is a minimally invasive procedure for facial rejuvenation that was introduced by Sulamanidze et al. [4] and has been recognized as a safe procedure with few major or minor complications.

However, some studies have pointed out that the facial rejuvenating effect after a procedure is lost over the course of long-term follow-up. Other studies have argued that thread lifting has limitations in terms of accomplishing the goals of facial rejuvenation, as it has little effectiveness and a short period of efficacy [5]. To resolve these concerns, other cosmetic procedures, such as fat grafts, laser treatment, and filler injections, have been combined with thread lifting [6,7].

In this study, we combined absorbable thread lifting with lipo- 
suction and compared the outcomes of this combined procedure with those of conventional absorbable thread lifting alone, focusing on both the rejuvenating effect and the duration of effectiveness.

\section{METHODS}

This study was conducted by reviewing the charts of patients who underwent thread lifting procedures between July 2013 and December 2015. Patients' medical records were reviewed retrospectively for information including age, clinical photographs, the procedures undertaken, and any complications.

Patients with a follow-up period of at least 6 months were enrolled in this study. To assess skin laxity, preoperative grading was performed using a 4-point scale (Table 1) [8].

Patients who had an active infectious disease that could alter wound healing and operation outcomes, had a history of psychiatric illness, or who had undergone previous facial procedures were excluded from this study.

The patients were divided into two groups according to the surgical procedure that was performed: thread lifting alone (group A) or thread lifting with liposuction (group B). The requirement for informed consent was waived, and this investigation was approved by the Institutional Review Board of Daegu Catholic University Medical Center (IRB No. CR-19-027).

\section{Surgical techniques}

Thread lifting with liposuction was performed using absorbable thread lifting and temporal anchoring. The thread used in this study was biodegradable polydioxanone minimal invasive nonsurgical thread (MINT Lift ML-1043; Hans Biomed, Seoul, Korea) and omega 41 (OV World, Seoul, Korea).

The preoperative design was made with the patient in an upright sitting position. We performed a preoperative pinch test and marked the boundary for liposuction depending on the amount of fatty tissue present. The exit points of the threads were between the following two lines: one from the lateral canthus to the submandibular angle, and the other a vertical line from the lateral canthus. The in- terval distance of each thread was $1 \mathrm{~cm}$. The route of the threads meant that the direction of lifting was almost vertical. The anchor site was $2 \mathrm{~cm}$ above the top of the ear around the superior temporal line, and, in some cases, careful shaving was performed to keep hairs from entering the anchoring site (Fig. 1).

After the design was made, liposuction was performed. A stab incision was applied by an infra-auricular incision or infra-jowl incision using a no. 11 scalpel blade, and $2.0 \mathrm{~mL}$ of lidocaine with 1:100,000

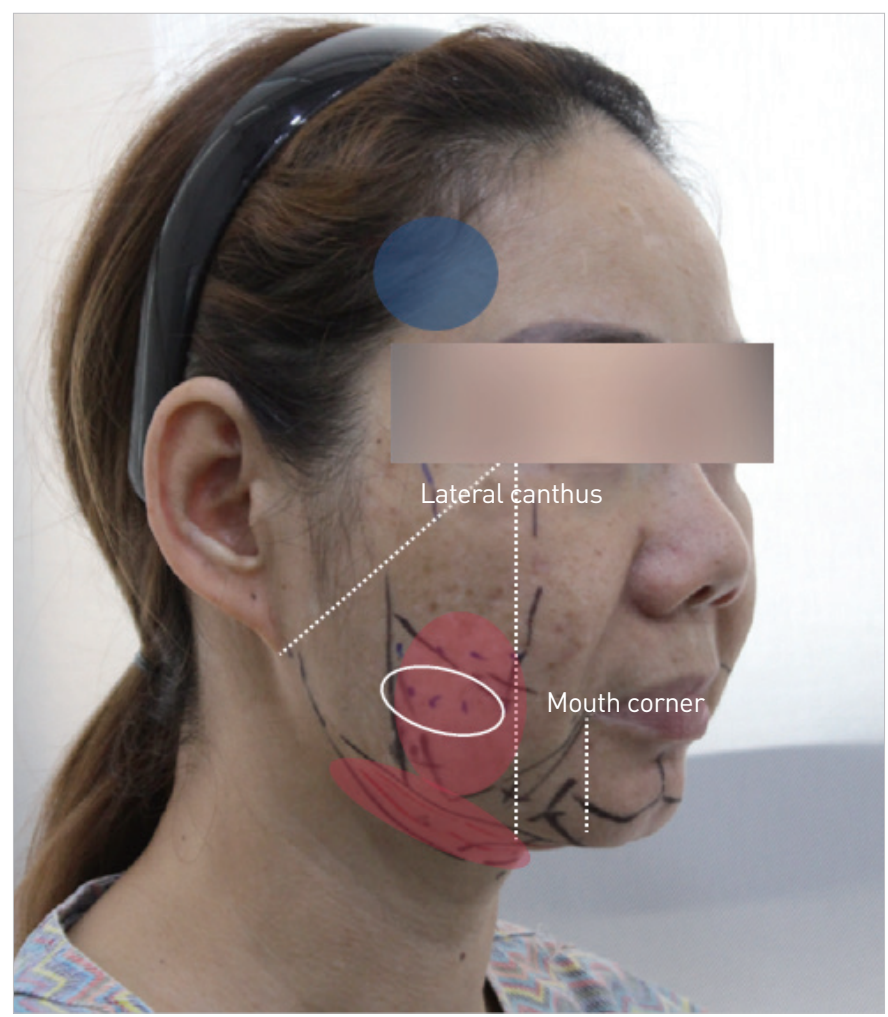

Fig. 1. A design for thread lifting with liposuction. The area for liposuction was marked depending on the amount of fatty tissue (red area). The anchoring site was around the superior temporal line (blue area). The exit point of the threads was in the area where liposuction was performed (white circle).

Table 1. Skin laxity grading scale [8]

\begin{tabular}{|c|c|c|}
\hline Grade & Descriptive parameter & Laxity \\
\hline 0 & None & None \\
\hline 1 & Mild & Localized to nasolabial folds \\
\hline 1.5 & Mild & Localized, nasolabial folds, early melolabial folds \\
\hline 2 & Moderate & Localized, nasolabial folds / melolabial folds, early jowls, early submental / submandibular \\
\hline 2.5 & Moderate & Localized, prominent nasolabial folds / melolabial folds, jowls and submental / submandibular \\
\hline 3 & Advanced & Prominent nasolabial folds / melolabial folds, jowls and submental / submandibular, early neck strands \\
\hline 3.5 & Advanced & Deep nasolabial folds / melolabial folds, prominent jowls and submental / submandibular, prominent neck strands \\
\hline 4 & Severe & Marked nasolabial folds / melolabial folds, jowls and submental / submandibular, neck redundancy and strands \\
\hline
\end{tabular}


epinephrine tumescent solution was then injected (approximately 20-30 mL on each side). After sufficient pretunneling through the incision site, a 14-gauge spatula-type cannula was inserted for liposuction. During this procedure, a pinch test was performed to prevent formation of an irregular contour due to excessive liposuction.

After liposuction, absorbable threads were anchored in the deep temporal fascia of the anchoring site near the superior temporal line using curved needles. A threaded long needle was inserted into the hole, passed through the subcutaneous layer, and taken out at the designed exit point. By repeating this maneuver, a total of four threads were inserted on each side. The excess threads at the exit point were gently pulled and then cut out. The intermediate postoperative dimple was resolved through manual compression.

For patients in group A, thread lifting using absorbable threads was done in the same manner, but the liposuction process was omitted, and the number of threads inserted on each side ranged from eight to 14 depending on the facial surface area and the degree of slack.

\section{Evaluation of outcomes}

In order to evaluate the outcomes of the procedures, the results were measured using the global aesthetic improvement scale (GAIS), which was used as a reference parameter (Table 2) [9]. The value recorded was determined independently by three plastic surgeons (DGH, YBL, and SEK). We also checked the patient's subjective satisfaction using a 4-point scale (1, unsatisfied; 2 , a little unsatisfied; 3 , a little satisfied; and 4, very satisfied).

\section{RESULTS}

\section{Epidemiology}

Of the patients who underwent thread lifting with or without liposuction between July 2013 to May 2015, 27 were observed for at least 6 months. Group A included 11 patients, and group B included 16 patients. All patients were female, and their mean age was 43.03 (43.72 in group A and 42.56 in group B). There was no significant difference in the mean skin laxity grade between each group (2.31 in group A and 2.15 in group B). The average follow-up period was 8 months.

\section{Surgical results}

The mean GAIS score for the thread lifting with liposuction group (group B) was 1.85, and the mean score for the thread lifting-only group (group A) was 2.54. The mean satisfaction score was 3.06 in group B, while the mean score in group A was 2.36 (Tables 3, 4). There were no major complications requiring additional treatment,

Table 2. The global aesthetic improvement scale

\begin{tabular}{|c|c|c|}
\hline Score & Degree & Description \\
\hline 1 & Very much improved & Optimal cosmetic result \\
\hline 2 & Much improved & Marked improvement of the appearance but not completely optimal \\
\hline 3 & Improved & Improvement of the appearance; better compared with the initial condition, but a touch-up is advised \\
\hline 4 & No change & The appearance remains substantially the same compared with the original condition \\
\hline 5 & Worsen & The appearance has worsened compared with the original condition \\
\hline
\end{tabular}

Table 3. Summary of the GAIS score and satisfaction score in the thread lifting-only group (groupA)

\begin{tabular}{|c|c|c|c|c|c|c|c|c|}
\hline Patient no. & Sex & $\begin{array}{l}\text { Age } \\
\text { (yr) }\end{array}$ & $\begin{array}{l}\text { Follow-up period } \\
\text { (mon) }\end{array}$ & $\begin{array}{l}\text { Skin laxity } \\
\text { grade }\end{array}$ & $\begin{array}{c}\text { GAIS by } \\
\text { surgeon } 1\end{array}$ & $\begin{array}{c}\text { GAIS by } \\
\text { surgeon } 2\end{array}$ & $\begin{array}{c}\text { GAIS by } \\
\text { surgeon } 3\end{array}$ & $\begin{array}{c}\text { Satisfaction } \\
\text { score }\end{array}$ \\
\hline 1 & $\mathrm{~F}$ & 33 & 7 & 1.5 & 2 & 3 & 2 & 2 \\
\hline 2 & $\mathrm{~F}$ & 34 & 7 & 2 & 3 & 4 & 4 & 2 \\
\hline 3 & $F$ & 46 & 12 & 2 & 3 & 3 & 3 & 1 \\
\hline 4 & $\mathrm{~F}$ & 46 & 8 & 3 & 4 & 3 & 2 & 2 \\
\hline 5 & $\mathrm{~F}$ & 47 & 7 & 3 & 3 & 2 & 3 & 2 \\
\hline 6 & $\mathrm{~F}$ & 53 & 6 & 3.5 & 3 & 3 & 3 & 1 \\
\hline 7 & $\mathrm{~F}$ & 54 & 6 & 2.5 & 4 & 3 & 4 & 2 \\
\hline 8 & $\mathrm{~F}$ & 50 & 7 & 2.5 & 2 & 3 & 3 & 3 \\
\hline 9 & $\mathrm{~F}$ & 26 & 9 & 1.5 & 1 & 3 & 1 & 4 \\
\hline 10 & $\mathrm{~F}$ & 41 & 11 & 2 & 2 & 1 & 1 & 4 \\
\hline 11 & $\mathrm{~F}$ & 51 & 14 & 2 & 1 & 1 & 1 & 3 \\
\hline
\end{tabular}

GAIS score: 1 (very much improved) to 5 (worsen); satisfaction score: 1 (unsatisfied) to 4 (very satisfied).

GAIS, global aesthetic improvement scale; $F$, female. 
Table 4. Summary of the GAIS score and satisfaction score in the thread lifting with liposuction group (group B)

\begin{tabular}{lccccccc}
\hline Patient no. & Sex & $\begin{array}{c}\text { Age } \\
\text { (yr) }\end{array}$ & $\begin{array}{c}\text { Follow-up period } \\
\text { (mon) }\end{array}$ & $\begin{array}{c}\text { Skin laxity } \\
\text { grade }\end{array}$ & $\begin{array}{c}\text { GAIS by } \\
\text { surgeon 1 }\end{array}$ & $\begin{array}{c}\text { GAIS by } \\
\text { surgeon 2 }\end{array}$ & $\begin{array}{c}\text { GAIS by } \\
\text { surgeon 3 }\end{array}$ \\
\hline 1 & F & 39 & 6 & 2 & 1 & 1 & 2 \\
2 & 26 & 7 & 1.5 & 1 & 2 & 2 & 2 \\
score
\end{tabular}

GAIS score: 1 (very much improved) to 5 (worsen); satisfaction score: 1 (unsatisfied) to 4 (very satisfied). GAIS, global aesthetic improvement scale; $F$, female.
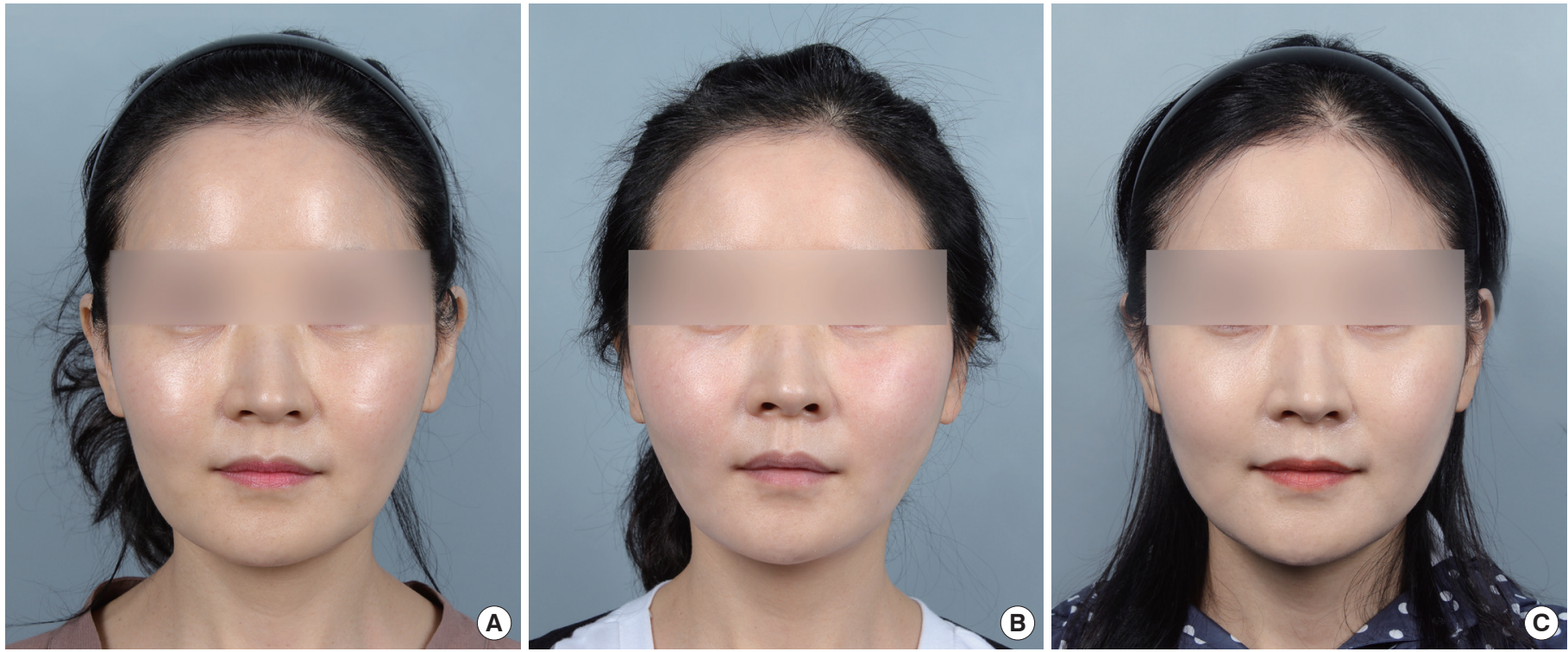

Fig. 2. A 33-year-old patient had complaints about the fullness of her lower face. She underwent thread lifting only, using 10 threads on each side. (A) Preoperative view. (B) At a 2-week follow-up, her lower face fullness had improved. (C) At a 3-month follow-up, she had persistent lower face fullness.

such as thread exposure, alopecia, or parotid gland injury. Five patients in group A and two patients in group B complained of ecchymosis. The ecchymosis lasted up to 3 weeks and did not require any treatment. All patients were able to return to their daily activities within 1 week of the procedure.

\section{DISCUSSION}

Due to the influence of mass media, recent standards of beauty have focused on facial youthfulness. This trend, in combination with the increasing life expectancy, have led to a rapid increase in 

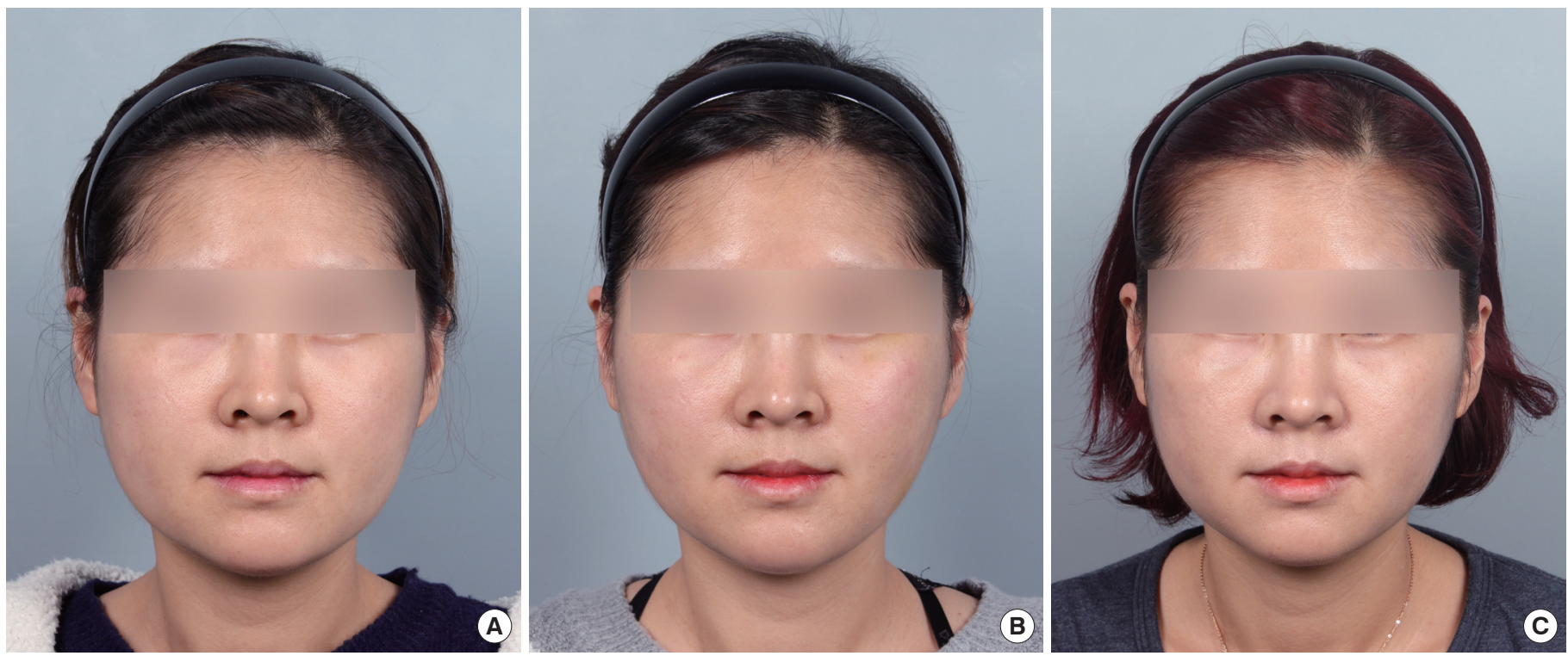

Fig. 3. A 34-year-old patient had complaints about a jowly lower face. She underwent thread lifting only, using 10 threads on each side. (A) Preoperative view. (B) At a 1-month follow-up, her jowly lower face had improved. (C) At a 4-month follow-up, she still had lower face jowls.
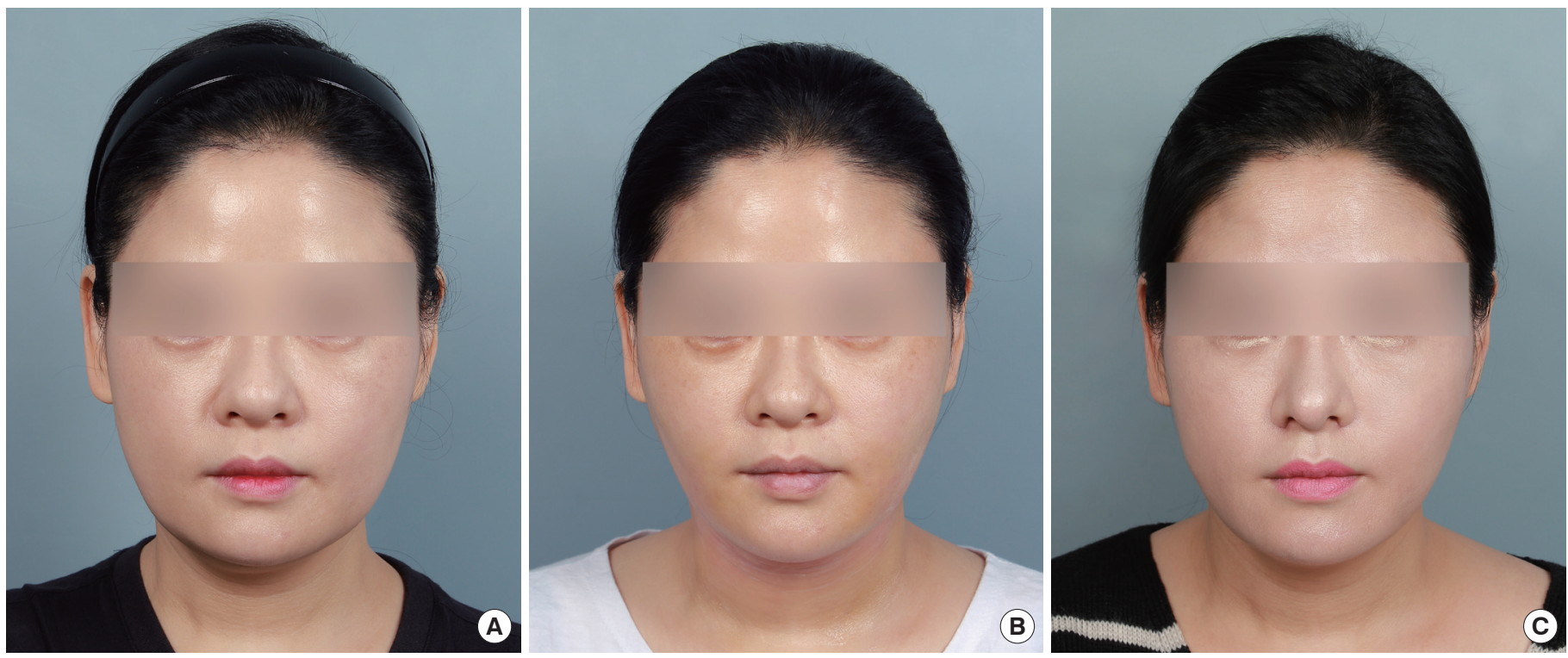

Fig. 4. A 28-year-old patient had complaints about lower face fullness. She underwent thread lifting using 4 threads on each side with liposuction. (A) Preoperative view. (B) A 1-week follow-up shows improved lower face fullness. (C) At a 7-month follow-up, the facial rejuvenating effect remained.

the number of operations and procedures related to facial rejuvenation $[1,2]$. In recent years, minimally invasive facial rejuvenation has come into the spotlight because of the low incidence of complications and the patient's rapid return to a normal daily routine.

Thread lifting is used to improve the appearance of aging in the lower face, jaw line, malar fat pad, and mid-face. Techniques of thread lifting for various applications, such as eyebrow lifting, neck lifting, and sub-mental lifting, have been developed [10]. However, a still-controversial disadvantage of the thread lifting procedure is its short-lasting facial rejuvenating effect.

Rachel et al. [11] reported that the incidence of early recurrence was $45 \%$ when an anchored barbed suture was used. They also argued that barbed sutures did not satisfy the patients' needs, although it was associated with lower morbidity and led to less downtime for the patients. Kaminer et al. [12] reported an average of 11.5 months of patient satisfaction after procedures using anchored 

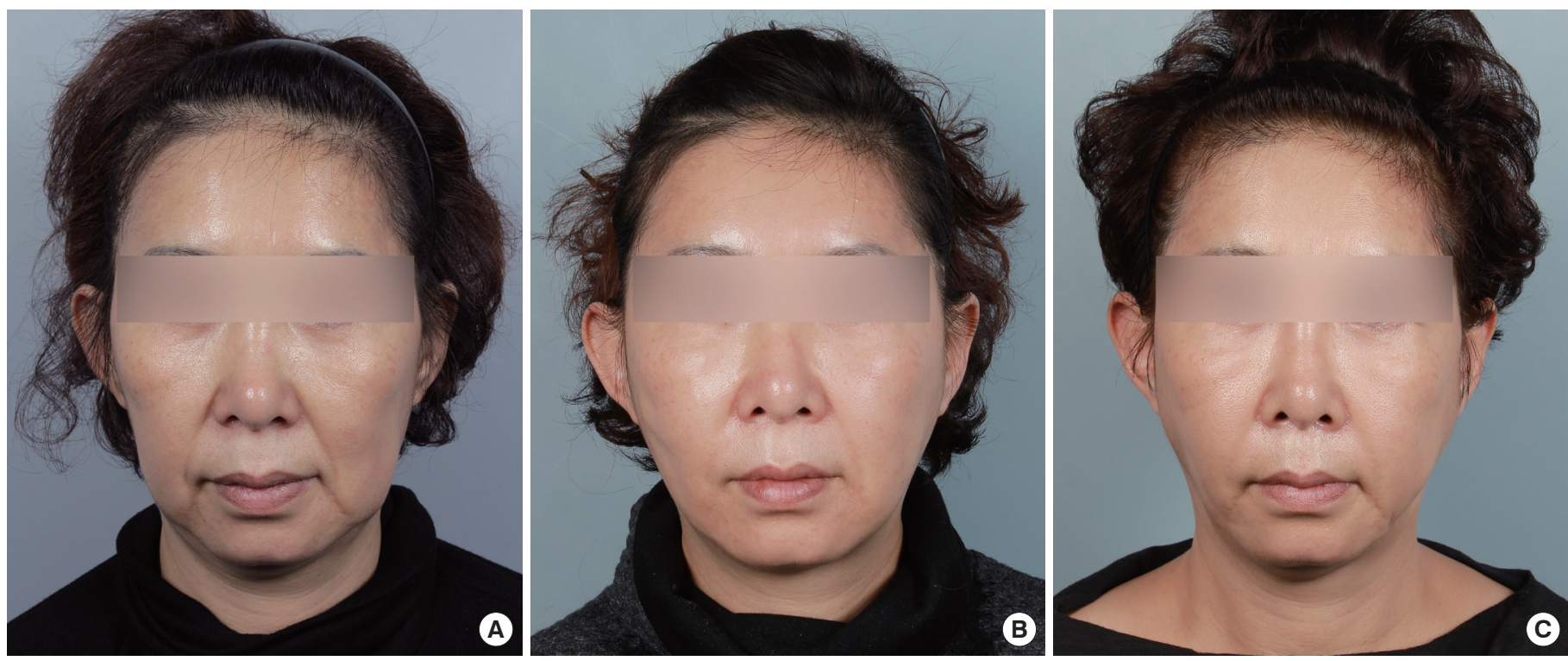

Fig. 5. A 54-year-old patient had complaints about lower face fullness and a jowly chin. She underwent thread lifting using 4 threads on each side with liposuction. (A) Preoperative view. (B) A 1-month follow-up view shows much improved lower facial aging. (C) A 7-month follow-up view shows that the lower facial rejuvenating effect persisted.

barbed sutures. In this study, the result of procedure with only thread lifting alone shows early recurrence (Fig. 2) and short-lasting facial rejuvenating effect (Fig. 3). Abraham et al. [5] suggested that thread lifting procedures have poor sustainability and are not suitable for facial rejuvenation because they do not cause a volumetric change in the facial area; instead, they only change the position of soft tissue in the superficial plane. In view of this, attempts have been made to improve the effectiveness of thread lifting by using other procedures in parallel, including fat grafts, filler injections, and laser treatment $[6,7]$.

Liposuction for facial rejuvenation is known to be useful. Shu and Lam [13] reported that liposuction using the tumescent technique was effective for facelifts in patients with thick and elastic skin, unlike that of Caucasians. When liposuction of the facial area is performed, amount of adipose tissue removed should not be more than $70 \%$. In the case of patients who are 50 or more years old, skin laxity should be reduced to $50 \%$, and the lip region should not be suctioned.

Before performing the procedures, we assessed patients' skin laxity using the skin laxity grading scale designed by Alexiades-Armenakas et al. [8] to avoid bias related to skin laxity. The mean score for preoperative skin laxity was 2.31 and 2.15 in group A and group $B$, respectively, and there was no significant difference between the two groups.

After these procedures, the mean score on the GAIS assigned by three plastic surgeons was 1.85 for group B, corresponding to better results than were obtained for group A (2.54). Furthermore, patients' satisfaction scores were higher in the combined procedure group than in the control group. Thus, the GAIS outcomes corresponded to the patients' subjective satisfaction.

As Abraham et al. [5] argued, thread lifting alone cannot induce volumetric change and has less efficacy for facial rejuvenation, but this weak point could be supplemented and resolved by combining thread lifting with a liposuction procedure. In our study, the result of thread lifting combined with liposuction shows good sustainability (Fig. 4) and improved effectiveness of thread lifting (Fig. 5).

In liposuction procedures, when the fatty layer is infused with a tumescent solution, the fat cells are broken apart and emulsify into the infusion fluid. These emulsified fatty cells can be easily removed, causing not only a volumetric reduction, but also shrinkage and tightening in the tissue layer during the healing process.

Liposuction also has complications, such as infection, postoperative subcutaneous panniculitis-like reaction, and skin retraction [14]. However, in this study, patients who underwent thread lifting with liposuction complained only of self-resolving problems, such as ecchymosis, and there were no complications requiring specific treatments. Thus, thread lifting combined with liposuction appears to be minimally invasive and has few complications, although it is necessary to investigate this issue further with a larger number of patients.

Throughout the course of the healing process, thread lifting combined with liposuction resulted in better clinical outcomes than thread lifting alone, and showed effectiveness over a long-term follow-up of more than 6 months.

However, to enable a more accurate comparison, a further study should be designed with more subjects, and such a study would 
preferably incorporate an objective evaluation technique using a recently developed skin diagnosis system in addition to the GAIS score [15].

In conclusion, patients who underwent thread lifting combined with liposuction showed greater satisfaction and better GAIS scores than those who received thread lifting only, even after a long follow-up period.

\section{NOTES}

\section{Conflict of interest}

No potential conflict of interest relevant to this article was reported.

\section{Ethical approval}

The study was approved by the Institutional Review Board of Daegu Catholic University Medical Center (IRB No. CR-19-027) and performed in accordance with the principles of the Declaration of Helsinki.

\section{Patient consent}

The patients provided written informed consent for the publication and the use of their images.

\section{ORCID}

Kwang Il Bae https://orcid.org/0000-0003-4496-2159

Dong Gil Han https://orcid.org/0000-0001-7922-5859

Sung-Eun Kim https://orcid.org/0000-0002-3413-7094

Young Bae Lee https://orcid.org/0000-0001-6879-0094

\section{REFERENCES}

1. Donofrio LM. Fat distribution: a morphologic study of the aging face. Dermatol Surg 2000;26:1107-12.

2. Yousif NJ. Changes of the midface with age. Clin Plast Surg 1995;22: 213-26.
3. Holliday R, Elfving-Hwang J. Gender, globalization and aesthetic surgery in South Korea. Body Soc 2012;18:58-81.

4. Sulamanidze M, Sulamanidze G, Vozdvizhensky I, et al. Avoiding complications with Aptos sutures. Aesthet Surg J 2011;31:863-73.

5. Abraham RF, DeFatta RJ, Williams EF 3rd. Thread-lift for facial rejuvenation: assessment of long-term results. Arch Facial Plast Surg 2009; 11:178-83.

6. Williams EF 3rd, Smith SP Jr. Minimally invasive midfacial rejuvenation: combining thread-lift and lipotransfer. Facial Plast Surg Clin North Am 2007;15:209-19.

7. Holcomb JD. Laser-assisted facelift. Facial Plast Surg 2014;30:405-12.

8. Alexiades-Armenakas M, Rosenberg D, Renton B, et al. Blinded, randomized, quantitative grading comparison of minimally invasive, fractional radiofrequency and surgical face-lift to treat skin laxity. Arch Dermatol 2010;146:396-405.

9. Narins RS, Brandt F, Leyden J, et al. A randomized, double-blind, multicenter comparison of the efficacy and tolerability of Restylane versus Zyplast for the correction of nasolabial folds. Dermatol Surg 2003;29: 588-95.

10. Yongtrakul P, Sirithanabadeekul P, Siriphan P. Thread lift: classification, technique, and how to approach to the patient. World Acad Sci Eng Technol 2016;10:558-66.

11. Rachel JD, Lack EB, Larson B. Incidence of complications and early recurrence in 29 patients after facial rejuvenation with barbed suture lifting. Dermatol Surg 2010;36:348-54.

12. Kaminer MS, Bogart M, Choi C, et al. Long-term efficacy of anchored barbed sutures in the face and neck. Dermatol Surg 2008;34:1041-7.

13. Shu T, Lam SM. Liposuction and lipotransplants for facial rejuvenation in the Asian patient. Int J Cosmet Surg Aesthet Dermatol 2003;5: $165-73$.

14. Hanke CW, Bernstein G, Bullock S. Safety of tumescent liposuction in 15,336 patients: national survey results. Dermatol Surg 1995;21:45962.

15. Kang MS, Shin JS, Nam SM, et al. Evaluation of elastic lift for facial rejuvenation. Arch Aesthetic Plast Surg 2016;22:20-7. 